\title{
A Novel Approach to General Linearly Constrained Adaptive Arrays
}

\author{
Byong Kun Chang ${ }^{1 *}$, Tae Yeon Kim², and Yong Kwon Lee ${ }^{3}$, Member, KIICE \\ ${ }^{1}$ Department of Electrical Engineering, University of Incheon, Incheon 406-772, Korea \\ ${ }^{2}$ Advanced Technology Inc., Incheon 406-840, Korea \\ ${ }^{3}$ Namyang Inc., Ansan 425-100, Korea
}

\begin{abstract}
A novel approach to general linearly constrained adaptive arrays is presented to improve the nulling performance in coherent and noncoherent environments. The narrowband and broadband linearly constrained adaptive arrays are implemented to examine the array performance. It is shown that the proposed approach performs better than the conventional adaptive arrays and the nulling performance depends on the gain factor for the desired response.
\end{abstract}

Index Terms: Adaptive array, Sensor, Linear constraint, Narrowband, Broadband, Coherent/noncoherent, Nulling performance

\section{INTRODUCTION}

In the linearly constrained adaptive array [1], the desired signal is estimated such that the array weights are updated iteratively by the linearly constrained least mean square (LMS) adaptive algorithm. It has been shown that the algorithm performs well if the desired signal and the interference signals are uncorrelated. It was shown that the signal interaction inherent in the algorithm causes partial cancellation of the desired signal in the array output even though the desired signal and the interference signals are uncorrelated [2]. If the interference signals are correlated with the desired signal, the signal interaction causes the desired signal to be partially or totally cancelled in the array output depending on the extent of correlation between the desired signal and the interference patterns.

To avoid the signal cancellation phenomenon, a variety of methods [2-9] have been proposed, such as a masterslave type array processor [2], a spatial smoothing approach
[3], and an alternate mainbeam nulling method $[4,5]$.

In this paper, a novel approach to the general linearly constrained adaptive array [1] is presented to improve the nulling performance of the conventional linearly constrained adaptive arrays in coherent and noncoherent environments. The desired response is formed as the output of the conventional beamformer weighted by a gain factor. The input signals to the conventional beamformer are given by those to the adaptive array. The narrowband and broadband adaptive arrays are implemented with coherent and noncoherent interference patterns.

The nulling performance is shown to depend on the value of the gain factor such that there exists an optimal value of the gain factor that minimizes the error power between the array output and the desired signal.

The proposed approach may be applied to the multipath environment in the area of wireless communications. 


\section{GENERAL LINEARLY CONSTRAINED NARROWBAND ADAPTIVE ARRAY}

The general linearly constrained narrowband adaptive array with Q sensor elements is shown in Fig. 1. The weights $\boldsymbol{w}_{n}, 1 \leq n \leq N$, are updated iteratively to minimize the power of the error output with a unit gain constraint in the look direction (i.e., the direction of the desired signal).

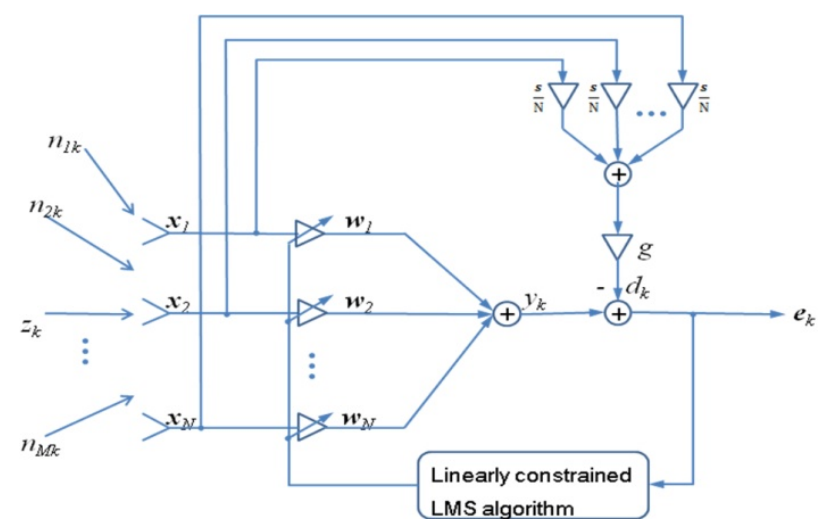

Fig. 1. General linearly constrained narrowband adaptive array. LMS: least mean square.

The optimization problem of finding an optimum weight vector for estimation of the desired signal is formulated as

$$
\begin{aligned}
& \min E\left[e_{k}^{2}\right] \\
& \text { subject to } \boldsymbol{w}^{H} \mathbf{s}=1
\end{aligned}
$$

where the weight vector $\boldsymbol{w}$, and the steering vector $\boldsymbol{s}$ of the desired signal are given by

$$
\begin{aligned}
\boldsymbol{w} & =\left[\begin{array}{llll}
w_{1} & w_{2} & \cdots & w_{N}
\end{array}\right]^{T} \\
\boldsymbol{s} & =\left[\begin{array}{llll}
1 e^{-j \beta \tau_{0}} & e^{-j 2 \beta \tau_{0}} & \cdots & e^{-j(N-1) \beta \tau_{0}}
\end{array}\right]^{T}
\end{aligned}
$$

where $\beta$ is the radian frequency of the desired signal, $\tau_{0}=d \sin \theta_{0} / v, \theta_{0}$ is the angle of incidene from the array normal, $d$ is inter-element spacing, $v$ is the signal propagation velocity, $k$ is an iteration index, and $\boldsymbol{E}$ and $\boldsymbol{T}$ denote expectation and transpose, respectively.

The error signal is given by

$$
e_{k}=y_{k}-d_{k}
$$

where the $y_{k}$ is given by

$$
y_{k}=\boldsymbol{w}^{H} \boldsymbol{x}_{k}
$$

and the desired response $d_{k}$ is

$$
d_{k}=g \frac{s^{H} x_{k}}{N}
$$

$$
\boldsymbol{x}_{k}=\left[\begin{array}{llll}
x_{k 1} & x_{k 2} & \cdots & x_{k N}
\end{array}\right]^{T},
$$

$g$ is a gain factor for the desired response, and $\boldsymbol{H}$ denotes the complex conjugate transpose. It is assumed that the angle of incidence for the desired signal is known a priori.

The iterative solution for the optimum weight vector may be found by the method of Lagrange multipliers [1], or what is called the general linearly constrained LMS algorithm, which is given by

$$
\boldsymbol{w}_{k+1}=\left[\boldsymbol{I}-\frac{s s^{H}}{N}\right]\left[\boldsymbol{w}_{k}-\mu e_{k}^{*} \boldsymbol{x}_{k}\right]+\frac{\boldsymbol{s}}{N}
$$

Where

$$
\boldsymbol{w}_{k}=\left[\begin{array}{llll}
w_{k 1} & w_{k 2} & \cdots & w_{k N}
\end{array}\right]^{T},
$$

$\boldsymbol{\mu}$ is convergence parameter, $\boldsymbol{I}$ is the $\boldsymbol{N} \times \boldsymbol{N}$ identity matrix, and $*$ denotes complex conjugate.

The weight vector $\boldsymbol{w}_{k}$ is updated iteratively to find a suboptimal solution of the optimization problem in (1). It is shown that the suboptimal solution depends on the gain factor $\boldsymbol{g}$ in the desired response path in Fig. 1.

\section{GENERAL LINEARLY CONSTRAINED BROADBAND ADAPTIVE ARRAY}

A linearly constrained broadband adaptive array is shown to cancel out the desired signal in a coherent environment [2]. To prevent the signal cancellation phenomenon, a general linearly constrained broadband adaptive array is proposed. The general linearly constrained broadband adaptive array with $N$ sensor elements followed by $\boldsymbol{L}$ taps per element is shown in Fig. 2.

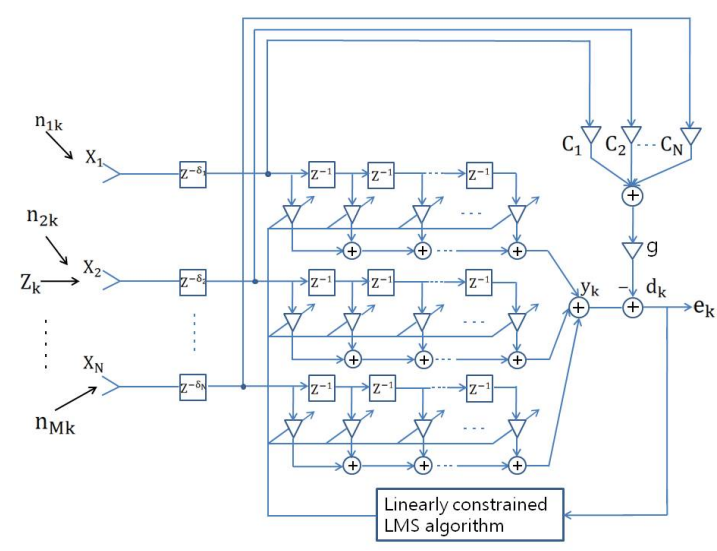

Fig. 2. General linearly constrained broadband adaptive array. LMS: least mean square. 
The desired response $d_{k}$ is produced as the output of the conventional beamformer scaled by the gain factor $g$.

To minimize the mean square error output with a unit gain constrained in the look direction, the weights are adjusted iteratively by the general linearly constrained LMS algorithm [1] as follows.

$$
\begin{aligned}
& \boldsymbol{w}_{0}=\left(\boldsymbol{C}^{\mathrm{T}} \boldsymbol{C}\right)^{-1} \boldsymbol{F} \\
& \boldsymbol{w}_{k+1}=\boldsymbol{P}\left[\boldsymbol{w}_{k}-\mu e_{k} \boldsymbol{x}_{k}\right]+\boldsymbol{F}
\end{aligned}
$$

where $C$ is an $N \boldsymbol{L} \times \boldsymbol{L}$ constraint matrix, $\boldsymbol{F}$ is given by

$$
\boldsymbol{F}=\boldsymbol{C}\left(\boldsymbol{C}^{\mathrm{T}} \boldsymbol{C}\right)^{-1} \boldsymbol{f}
$$

$\boldsymbol{f}$ is an $\boldsymbol{L} \times 1$ constraint vector, $\boldsymbol{P}$ is a projection matrix that projects any vector onto the constraint subspace and is given by

$$
\boldsymbol{P}=\boldsymbol{I}-\boldsymbol{C}\left(\boldsymbol{C}^{\mathrm{T}} \boldsymbol{C}\right)^{-1} \boldsymbol{C}^{\mathrm{T}} .
$$

It is worth noting that the constraint subspace is the orthogonal complement of the column space of $\boldsymbol{C}$.

The array weights are updated such that the desired signal is estimated in a least mean square sense such that the array output power is minimized while maintaining the unit gain in the look direction.

\section{SIMULATION RESULTS}

\section{A. Narrowband Case}

A narrowband linear array with 7 equispaced sensor elements is employed to examine the performance of the proposed method. The desired signal is assumed to be a sinusoid incident at the array normal. The cases for one and two coherent signal, and one noncoherent signal interference are simulated. The frequency of the noncoherent interferencen is assumed to be half that of the desired signal in the noncoherent case. The incoming signals are assumed to be plane waves. The nulling performances are compared with respect to the gain factor $\boldsymbol{g}$ and the conventional linearly constrained adaptive array proposed by Frost [1]. The convergence parameter $\boldsymbol{\mu}$ is assumed to be 0.001 .

\section{1) One Coherent Signal Interference Case}

It is assumed that a coherent interference is incident at $30^{\circ}$ from the array normal. The variation of the error power between the array output and the desired signal is displayed in terms of the gain factor $\boldsymbol{g}$ in Fig. 3. The optimum value of $\boldsymbol{g}$ that yields the minimum error power is shown to be 0.331 .
The comparison of the array performance for $\boldsymbol{g}=0.331,0.01$, and the conventional linearly constrained adaptive array proposed by Frost [1] are shown in Figs. 4 and 5 with respect to the array output and the desired signal for $k=1-1,000$ and 29,001-30,000 samples, respectively. It is demonstrated that the case for $\boldsymbol{g}=0.031$ performs best while the case for $\boldsymbol{g}$ $=0.01$ performs better than Frost's. It is shown that the desired signal of Frost's array disappears (i.e., is cancelled out) in the steady state array output for $29,001 \leq \mathrm{k} \leq 30,000$. The beam patterns are shown in Fig. 6, in which the case for $\boldsymbol{g}=0.031$ makes a deep null $(-51 \mathrm{~dB})$ in the direction of the interference pattern $\left(30^{\circ}\right)$.

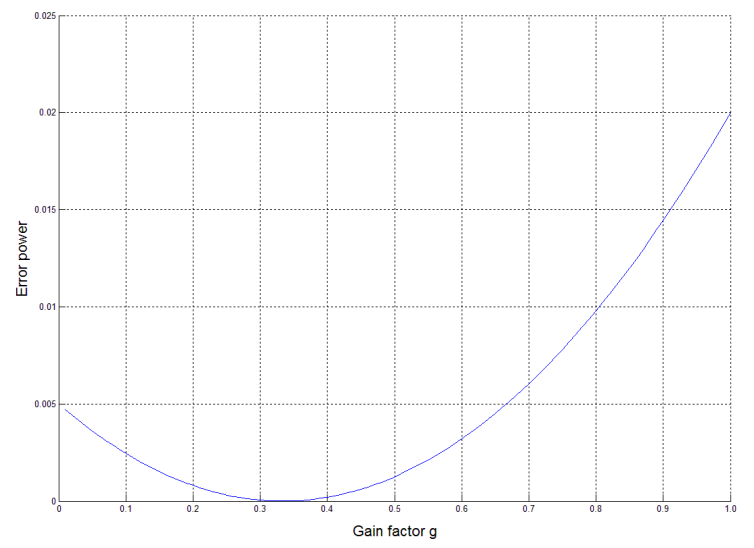

Fig. 3. Variation in the power of the error signal in terms of the gain factor for one coherent signal interference case.
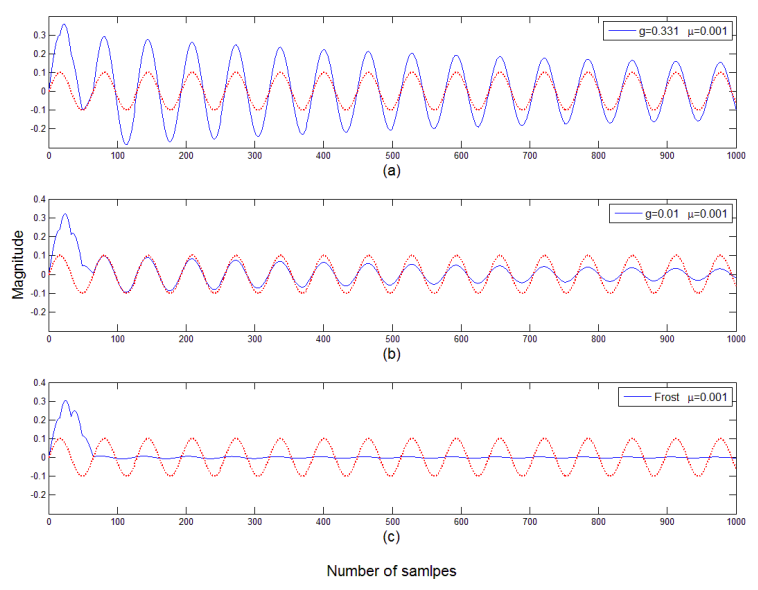

Fig. 4. Comparison of the array output and desired signal for one coherent signal interference case for $1 \leq k \leq 1,000$. 

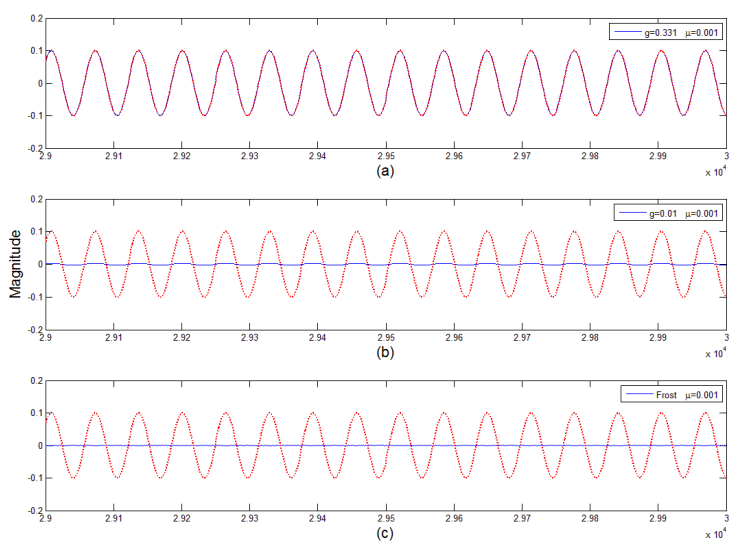

Number of samlpes

Fig. 5. Comparison of the array output and desired signal for one coherent signal interference case for $29,001 \leq k \leq 30,000$.

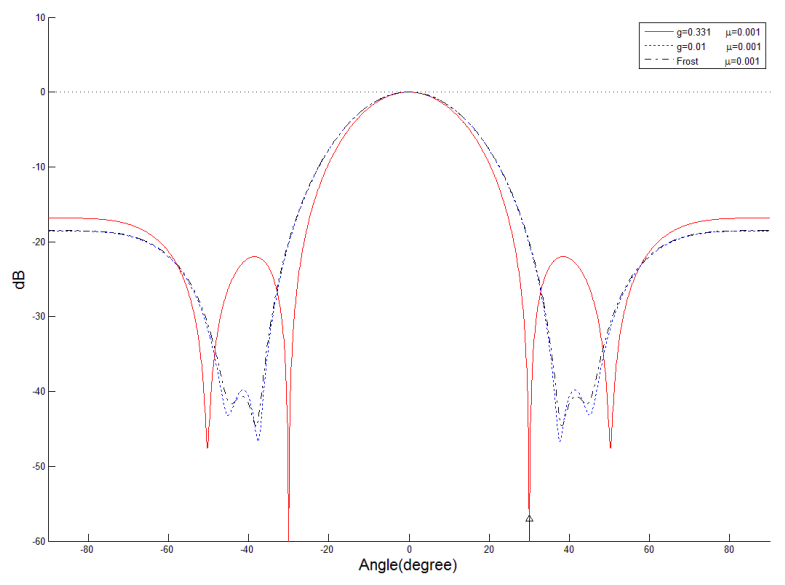

Fig. 6. Comparison of the beam patterns for one coherent signal interference case.

\section{2) Two Coherent Signal Interference Case}

It is assumed that two coherent signal interferences are incident at $-54.3^{\circ}$ and $57.5^{\circ}$ from the array normal. The variation in the error power between the array output and the desired signal is displayed in Fig. 7. The optimum value of $\boldsymbol{g}$ is shown to be 0.632 . The comparison of the array performances for $\boldsymbol{g}=0.632,0.01$, and the conventional linearly constrained adaptive array proposed by Frost are shown in Figs. 8 and 9 with respect to the array output and the desired signal for $\mathrm{k}=1-1,000$ and 29,001-30,000 samples, respectively. It is shown that the case for $\boldsymbol{g}=0.632$ performs best while the case for $\boldsymbol{g}=0.01$ performs similarly to Frost's. The beam patterns are shown in Fig. 10, in which the case for $\boldsymbol{g}=0.632$ makes two deep nulls $(-36.6 \mathrm{~dB}$ and $-30.4 \mathrm{~dB})$ at two incident angles $\left(-54.3^{\circ}\right.$ and $\left.57.5^{\circ}\right)$ of the coherent interference patterns while the gains for Frost's are $-21.0 \mathrm{~dB}$ and $-21.8 \mathrm{~dB}$ and the gains for $\boldsymbol{g}=0.01$ are $-22.7 \mathrm{~dB}$ and $-25.1 \mathrm{~dB}$, respectively.

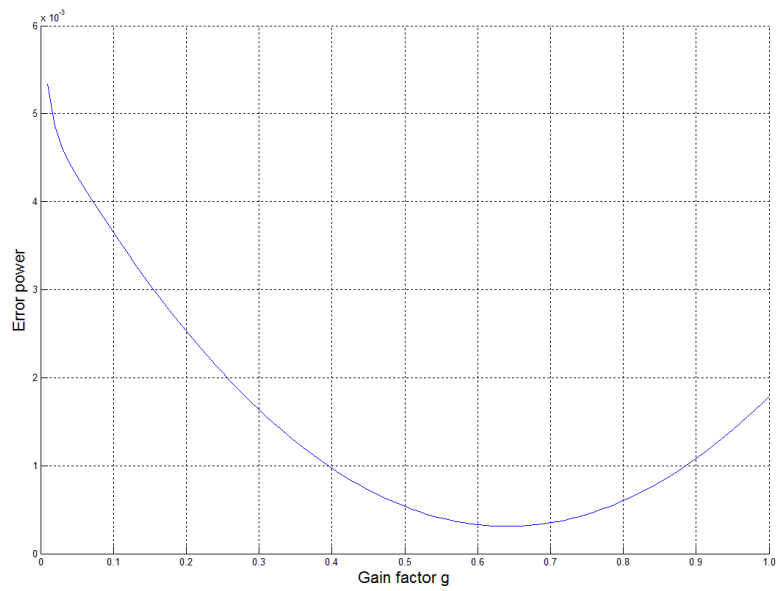

Fig. 7. Variation in the power of the error signal in terms of the gain factor for two coherent signal interference case.
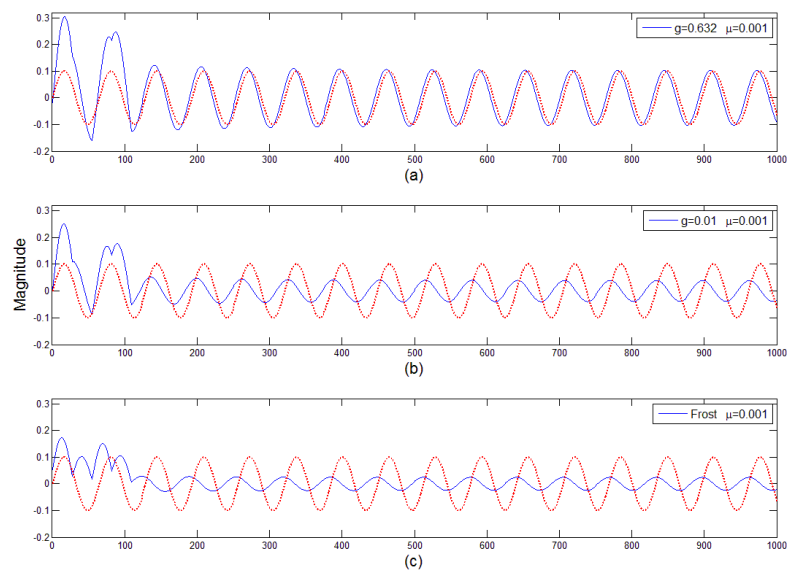

Number of sampes

Fig. 8. Comparison of the array output and desired signal for two coherent signal interference case for $1 \leq k \leq 1,000$.
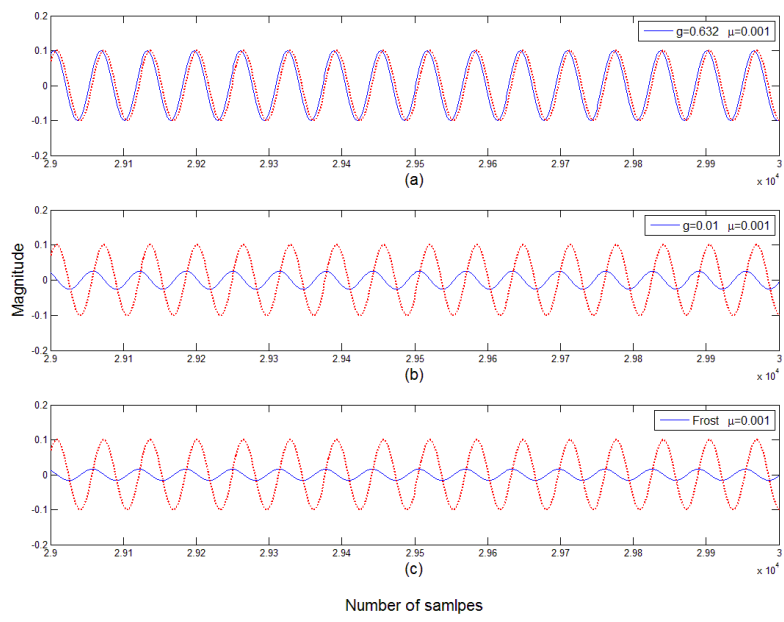

Fig. 9. Comparison of the array output and desired signal for two coherent signal interference case for $29,001 \leq k \leq 30,000$. 


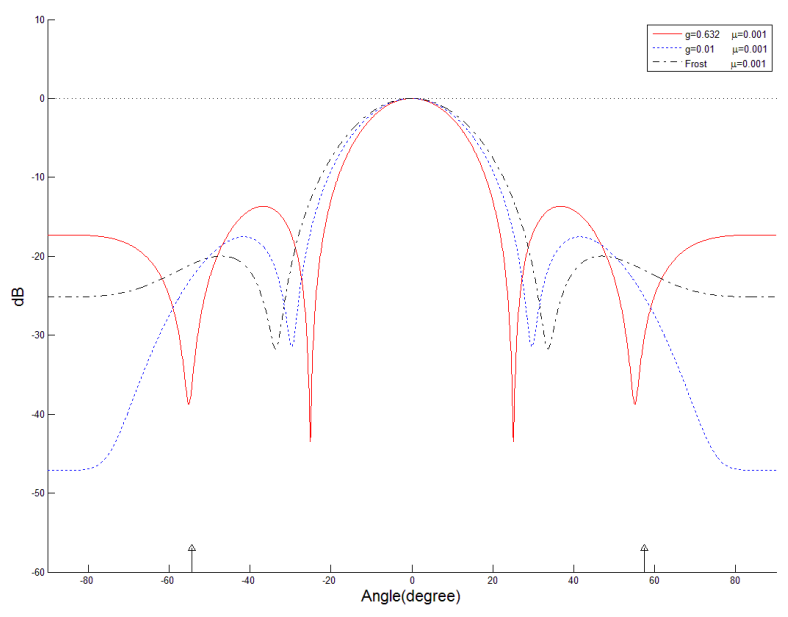

Fig. 10. Comparison of the beam patterns for the two-coherent interference case.

\section{3) One Noncoherent Signal Interference Case}

It is assummed that a noncoherent interference is incident at $-64.9^{\circ}$ from the array normal. The variation of the error power between the array output and the desired signal is displayed in Fig. 11. The optimum value of $\boldsymbol{g}$ is shown to be 0.0 . The comparison of the array performance for $\boldsymbol{g}=0.01$, 1.0 , and the conventional linearly constrained adaptive array proposed by Frost are shown in Figs. 12 and 13 with respect to the array output and the desired signal for $k=1-1,000$ and 29,001-30,000 samples, respectively. It is shown that the case for $\boldsymbol{g}=0.01$ and Frost's array[1] yield similar performances while both of them perform better than the case for $\boldsymbol{g}=1.0$. The beam patterns are shown in Fig. 14, in which the case for $\boldsymbol{g}=0.01$ and Frost's array yield similar gains $(-18.6 \mathrm{~dB})$ at the angle of incidence of the interference.

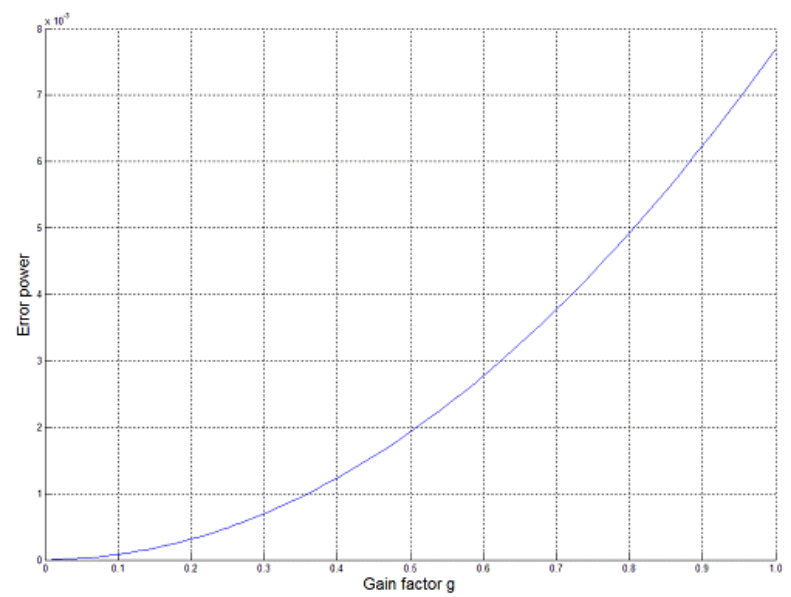

Fig. 11. Variation in the power of the error signal in terms of the gain factor for the one noncoherent interference case.
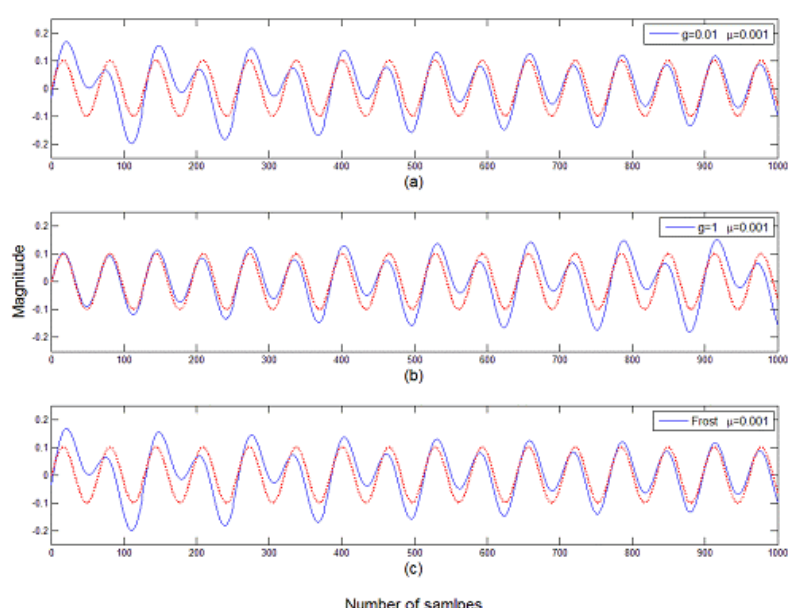

Fig. 12. Comparison of the array output and desired signal for the one noncoherent interference case for $1 \leq k \leq 1,000$.
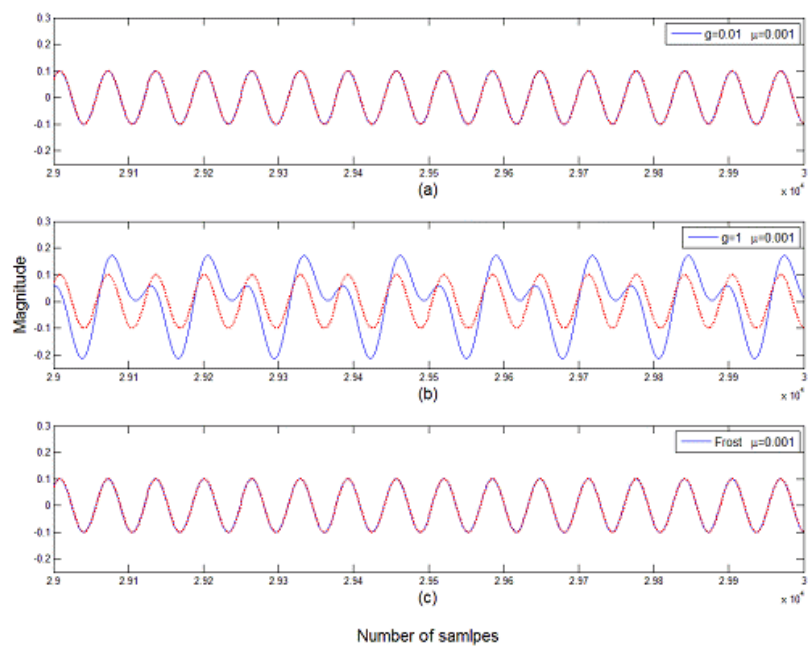

Fig. 13. Comparison of the array output and desired signal for the one noncoherent interference case for $29,001 \leq k \leq 30,000$.

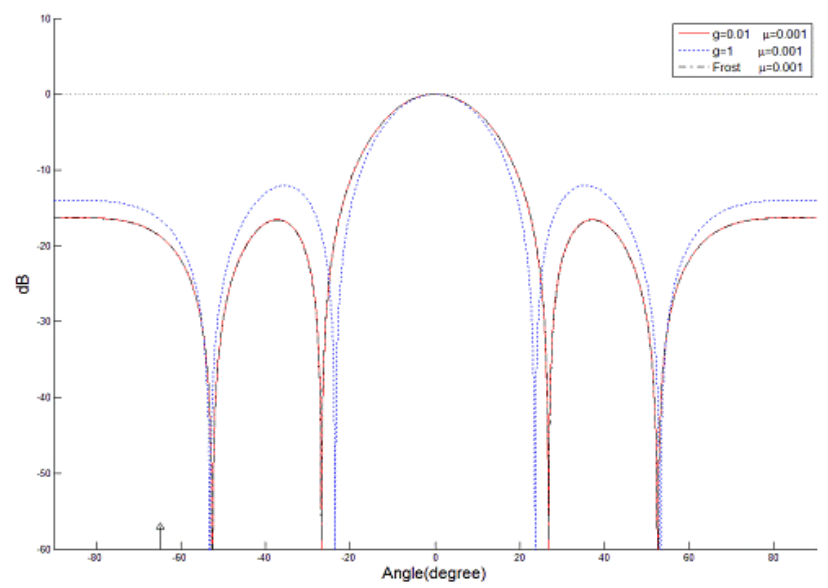

Fig. 14. Comparison of the beam patterns for the one noncoherent signal interference case. 


\section{B. Broadband Case}

A broadband linear adaptive array with 5 sensor elements and 3 weights per element is simulated with incoming broadband signals. The broadband desired signal is generated by passing a white Gaussian random signal through a 4th-order Butterworth filter such that the bandwidth is $3 \mathrm{~Hz}$ with the upper and lower frequencies 8 $\mathrm{Hz}$ and $11 \mathrm{~Hz}$, respectively. The incoming signals are assumed to be plane waves. The cases for one and two coherent signal and one noncoherent signal interferences are simulated. The sampling frequency is $608 \mathrm{~Hz}$. The convergence parameter $\mu$ is assumed to be 0.0001 .

\section{1) One Coherent Interference Case}

It is assummed that a coherent interference is incident at $30^{\circ}$ from the array normal. The variation of the error power between the array output and the desired signal is displayed in Fig. 15. The optimum value of $g$ is shown to be 0.33 . The comparison of the array performance for $\boldsymbol{g}=$ 0.33 , the conventional linearly constrained adaptive array proposed by Frost and $\boldsymbol{g}=2.0$ are shown in Figs. 16 and 17 with respect to the array output and the desired signal for the $k=1-1,000$ and 29,001-30,000 samples, respectively. It is shown that the case for $\boldsymbol{g}=0.33$ performs best while Frost's performs better than the case for $\boldsymbol{g}=2.0$. The beam patterns are shown in Fig. 18, in which the case for $\boldsymbol{g}=0.33$ makes a deep null close to the interference direction.

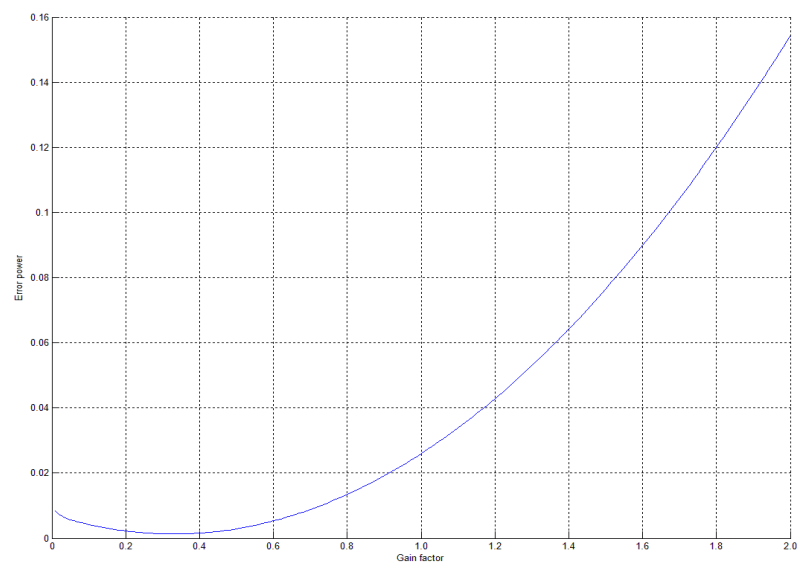

Fig. 15. Variation of the power of the error signal in terms of gain factor for one coherent interference case.
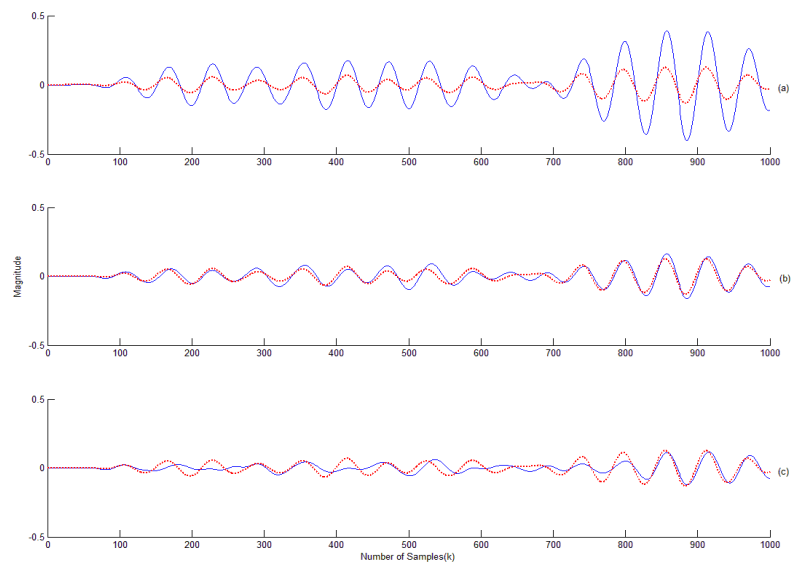

Fig. 16. Comparison of the array output (solid line) and desired signal (dotted line) for one coherent interference case; (a) $g=0.33$, (b) Frost, (c) $g=2$, for $1 \leq k \leq 1,000$
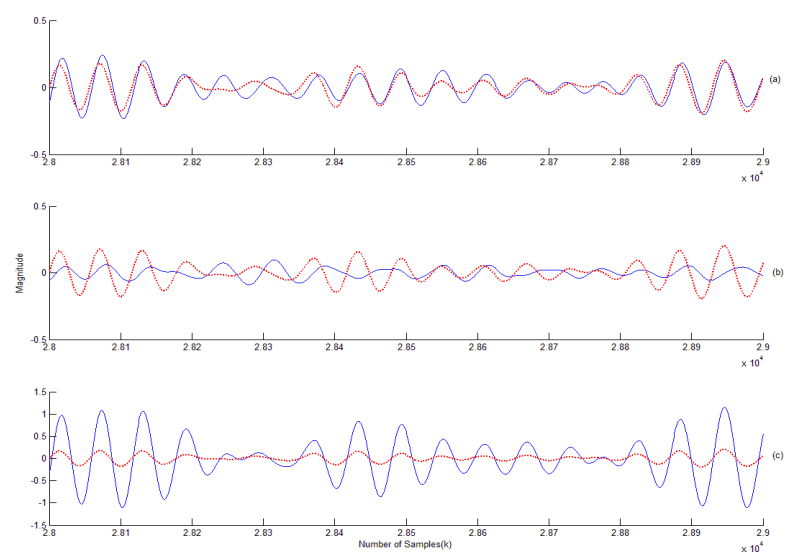

Fig. 17. Comparison of the array output (solid line) and desired signal (dotted line) for one coherent interference case; (a) $\mathrm{g}=0.33$, (b) Frost, (c) $\mathrm{g}=2$, for $29,001 \leq \mathrm{k} \leq 30,000$

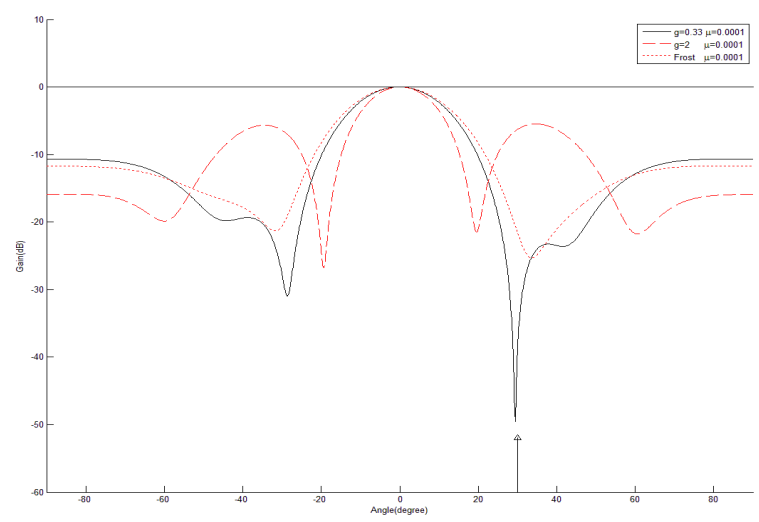

Fig. 18. Comparison of beam patterns for one coherent interference case at $30^{\circ}$. 


\section{2) Two Coherent Interference Case}

It is assummed that two coherent signal interference patterns are incident at $-54.3^{\circ}$ and $57.5^{\circ}$ from the array normal. The variation in the error power between the array output and the desired signal is displayed in Fig. 19. The optimum value of $\boldsymbol{g}$ is shown to be 0.29 . The comparison of the array performance for $\boldsymbol{g}=0.29$, the conventional linearly constrained adaptive array proposed by Frost, and $\boldsymbol{g}=2.0$ are shown in Figs. 20 and 21 with respect to the array output and the desired signal for the $\mathrm{k}=1-1,000$ and $29,001-30,000$ samples, respectively. It is shown that the case for $\boldsymbol{g}=0.29$ performs best while Frost's performs better than the case for $\boldsymbol{g}=0.01$. The beam patterns are shown in Fig. 22, in which the case for $\boldsymbol{g}=0.29$ makes two deep nulls around the two incident angles $-54.3^{\circ}$ and $57.5^{\circ}$ of the coherent interferences.

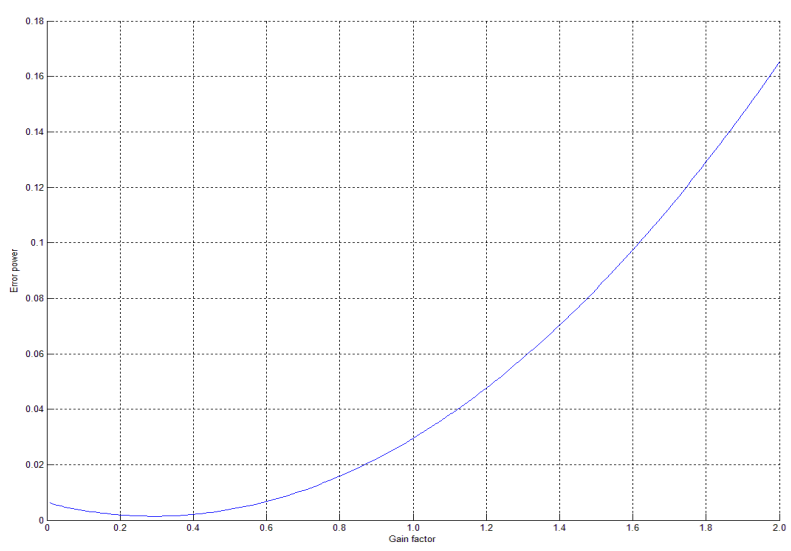

Fig. 19. Variation in the power of the error signal in terms of the gain factor for the two coherent signal interference case.
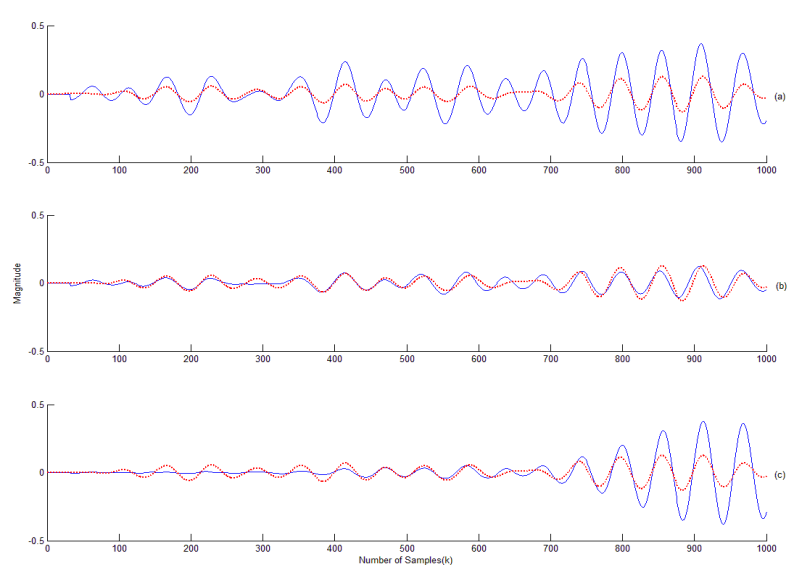

Fig. 20. Comparison of the array output (solid line) and desired signal (dotted line) for two coherent signal interference case; (a) $g=0.29$, (b) Frost, (c) $\mathrm{g}=2.0$, for $1 \leq \mathrm{k} \leq 1,000$.
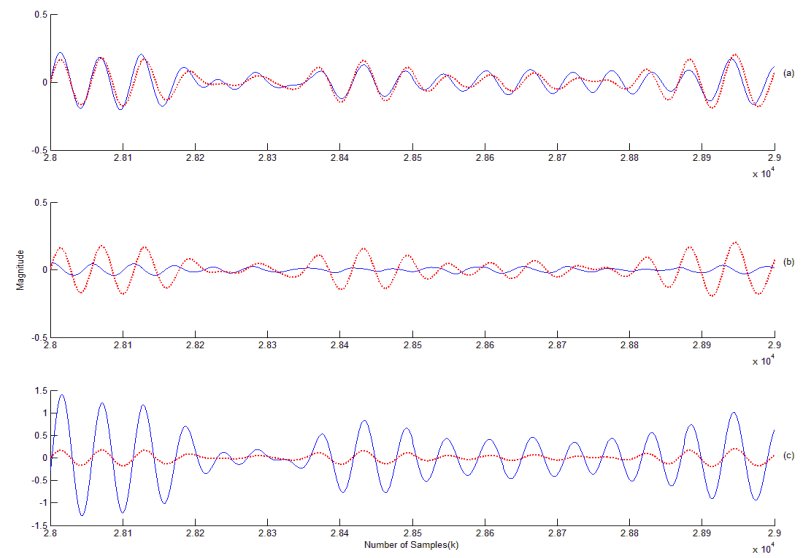

Fig. 21. Comparison of the array output (solid line) and desired signal (dotted line) for the two coherent signal interference case; (a) $g=0.29$, (b) Frost, (c) $g=2.0$, for $29,001 \leq k \leq 30,000$.

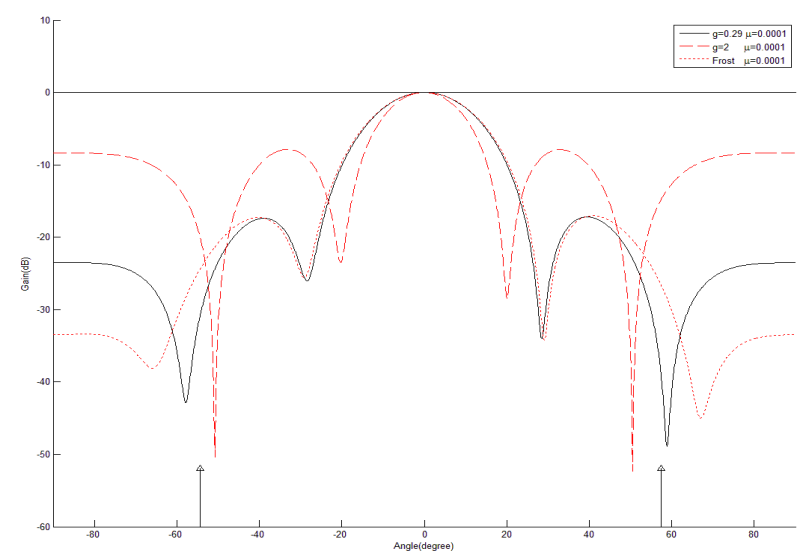

Fig. 22. Comparison of the beam patterns for the two coherent signal interference case at $-54.3^{\circ}, 57.5^{\circ}$.

\section{3) One Noncoherent Interference Case}

It is assumed that a noncoherent interference is incident at $-48.5^{\circ}$ from the array normal. The variation of the error power between the array output and the desired signal is displayed in Fig. 23. The optimum value of $\boldsymbol{g}$ is shown to be 0.09 . The comparison of the array performance for $\boldsymbol{g}=0.09$, the conventional linearly constrained adaptive array proposed by Frost, and $\boldsymbol{g}=2.0$ are shown in Figs. 24 and 25 with respect to the array output and the desired signal for the $k=1-1,000$ and 29,001-30,000 samples, respectively. It is shown that the case for $\boldsymbol{g}=0.09$ and Frost's array perform similarly, while both of them perform better than the case for $\boldsymbol{g}=2.0$. The beam patterns are shown in Fig. 26, in which the case for $\boldsymbol{g}=0.09$ and Frost's array yield a similar gain at the angle of incidene of the interference. 


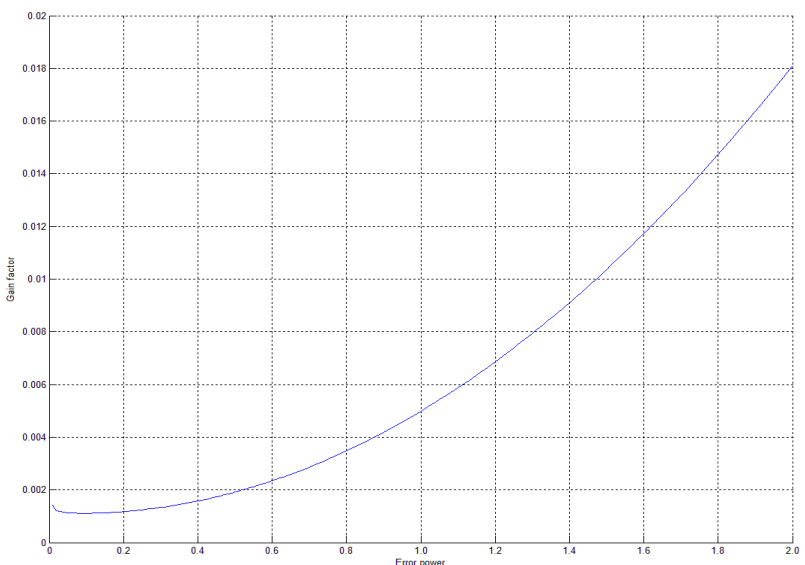

Fig. 23. Variation in the power of the error signal in terms of the gain factor for one noncoherent interference case.
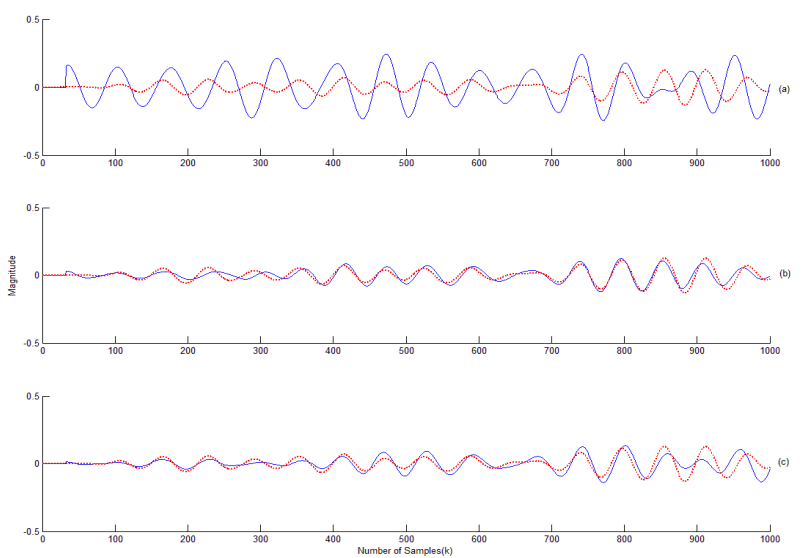

Fig. 24. Comparison of array output (solid line) and desired signal (dotted line) for one noncoherent interference case; (a) $g=0.09$, (b) Frost, (c) $\mathrm{g}=2.0$, for $1 \leq \mathrm{k} \leq 1,000$.
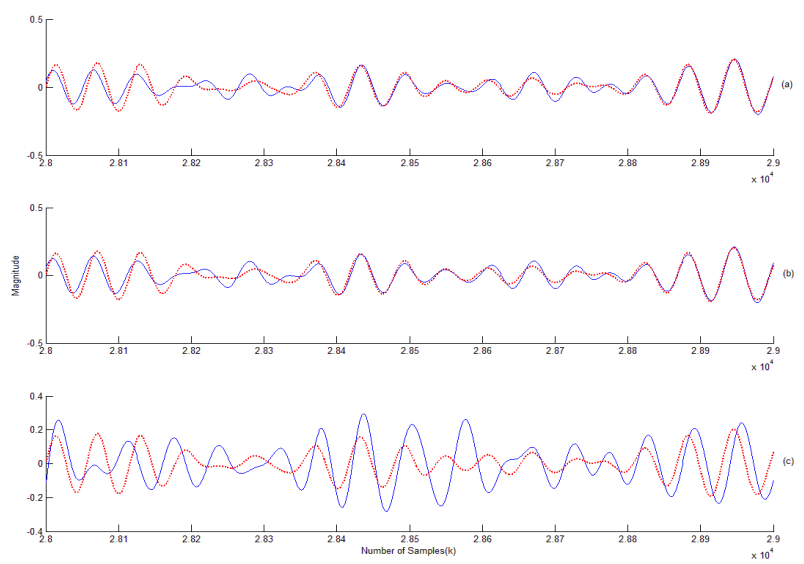

Fig. 25. Comparison of array output (solid line) and desired signal (dotted line) for one noncoherent interference case; (a) $g=0.09$, (b) Frost, (c) $g=2.0$, for $29,001 \leq k \leq 30,000$.

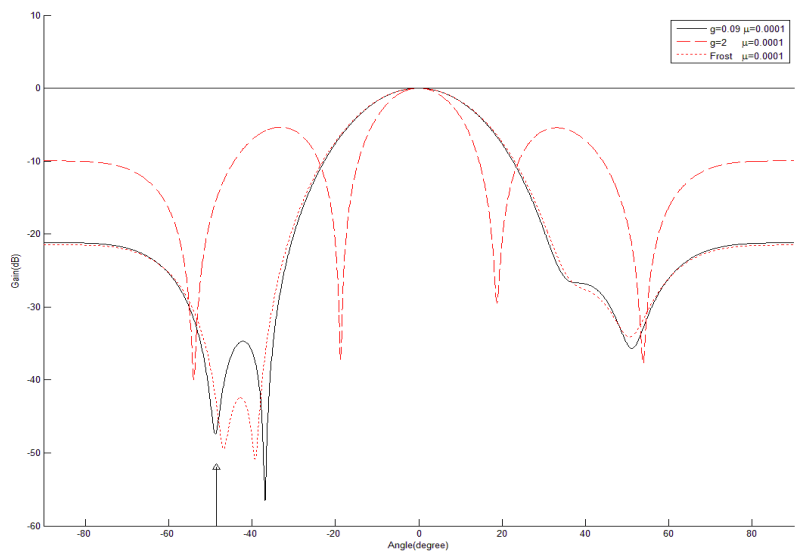

Fig. 26. Comparison of the beam patterns for the one noncoherent interference case at $-48.5^{\circ}$.

\section{CONCLUSIONS}

A general linearly constrained adaptive array is proposed and implemented to examine its performance in coherent and noncoherent signal environments. The performance of the proposed approach is examined with narrowband and broadband adaptive arrays in different signal environments.

In the narrowband case, it is shown that the proposed approach performs best at an optimal gain factor in a coherent environment while it performs similarly to Frost's array as the gain factor decreases in a noncoherent environment. In the broadband case, the proposed approach performs better at the optimal gain factor than does Frost's array in a coherent environment while it yields a similar performance to Frost's in a noncoherent environment.

\section{ACKNOWLEDGMENTS}

This work was supported by a University of Incheon Research Grant in 2010.

\section{REFERENCES}

[1] O. L. Frost, “An algorithm for linearly constrained adaptive array processing," Proceedings of the IEEE, vol. 60, no. 8, pp. 926-935, 1972.

[2] B. Widrow, K. Duvall, R. Gooch, and W. Newman, "Signal cancellation phenomena in adaptive antennas: Causes and cures," IEEE Transactions on Antennas and Propagation, vol. 30, no. 3, pp. 469-478, 1982.

[3] T. J. Shan and T. Kailath, "Adaptive beamforming for coherent signals and interference," IEEE Transactions on Acoustics, Speech and Signal Processing, vol. 33, no. 3, pp. 527-536, 1985. 
[4] B. K. Chang, C. D. Jeon, and D. H. Song, "Performance improvement in alternate mainbeam nulling by adaptive estimation of convergence parameters in linearly constrained adaptive arrays,' International Journal of Maritime Information and Communication Sciences, vol. 7, no. 3, pp. 392-398, 2009.

[5] B. K. Chang and C. D. Jeon, "Research for performance analysis of antenna arrays in basestation for GSM system," Journal of Korean Institute of Electromagnetic Engineering and Science, vol. 16, no. 7, pp. 661-775, 2005.

[6] B. K. Chang, T. Y. Kim, and Y. K. Lee, "Performance of general linearly constrained adaptive arrays," Proceedings of the 41th Korean Institute of Electrical Engineers Summer Conference, pp. 1937-2939, 2010.
[7] Y. L. Su, T. J. Shan, and B. Widrow, "Parallel spatial processing: a cure for signal cancellation in adaptive arrays," IEEE Transactions on Antenna and Propagation, vol. 34, no. 3, 1986.

[8] B. K. Chang, N. Ahmed, and D. H. Youn, "Fast convergence adaptive beamformers with reduced signal cancellation," Proceedings of the 22nd Asilomar Conference on Signals, Systems and Computers, Pacific Grove, CA, pp. 823-827, 1988.

[9] B. Widrow and S. D. Stearns, Adaptive Signal Processing, Englewood Cliffs, NJ: Prentice-Hall, 1985.

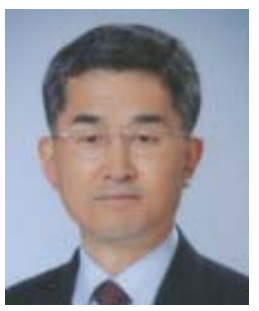

\section{Byong Kun Chang}

received a B.E. degree from the Dept. of Electronics Engineering of Yonsei University in 1975, an M.S. degree from the Dept. of Electrical and Computer Engineering of the University of lowa in 1985, and a Ph.D. from the Dept. of Electrical and Computer Engineering of the University of New Mexico in 1991. He is a Professor in the Dept. of Electrical Engineering of the University of Incheon. His research interests include adaptive signal processing, array signal processing, and microcomputer applications.

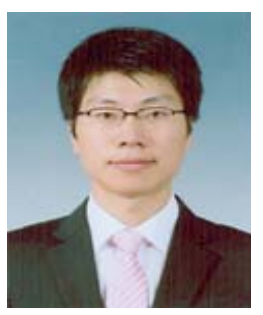

\section{Tae Yeon Kim}

received the B.E. and M.S. degrees from the Dept. of Electrical Engineering of the University of Incheon in 2009 and 2011, respectively, and is a senior engineer in the Research \& Development Dept. of ATI. Inc. His research interests include microcomputer applications and adaptive signal processing.

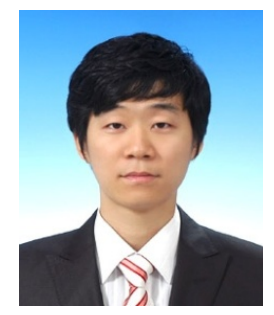

\section{Yong Kwon Lee}

received the B.E. and M.S. degrees from the Dept. of Electrical Engingeering of the University of Incheon in 2009 and 2011 , respectively, and he is a senior engineer in the Research \& Development Dept. of Namyang, Inc. His research interests include microcomputer applications and adaptive signal processing. 